\title{
Miscibility and Luminescence Properties of MEH-PPV/DPO-PPV Polyblends
}

\author{
Hsuan-Liang Chou ${ }^{1}$, King-Fu Lin ${ }^{1, *}$ and Ding-Chang Wang ${ }^{2}$ \\ ${ }^{1}$ Department of Material Science and Engineering, National Taiwan University, Taipei, 10617, Taiwan, \\ Republic of China \\ ${ }^{2}$ Ritekdisplay Corporation, No. 12, Kuanfu N. Rd., Hsin Chu Industrial Park, 30316, Taiwan, Republic of China \\ (*Author for correspondence; Tel.: +886-2-23928290; Fax: +886-2-23634562; E-mail: kflin@ccms.ntu.edu.tw)
}

Received 16 April 2005; accepted in revised form 31 May 2005

Key words: conjugated polymers, luminescence, polymer light-emitting diode, poly(2-methoxy-5-(2'-ethyl-hexyloxy)p-phenylenevinylene), polyblends

\begin{abstract}
Poly(2-methoxy-5-(2'-ethyl-hexyloxy)-p-phenylenevinylene) (MEH-PPV) and poly(2,3-diphenyl-5-octyl-p-phenylenevinylene) (DPO-PPV) are basically immiscible as verified by fluorescence spectroscopy and differential scanning calorimetry. This immiscibility results in insufficient energy transfer from DPO-PPV to MEH-PPV in photoluminescence. A vertically segregated structure with DPO-PPV green domains dispersing on the MEH-PPV-rich matrix was observed in a spin-cast film by two-photon excitation microscopy. The turn-on voltages for electroluminescence (EL) of polyblends were lower than those of their individual pristine polymers, while their EL quantum efficiencies were higher. Because both the highest occupied molecular orbital and the lowest unoccupied molecular orbital levels of MEH-PPV are higher than those of the DPO-PPV, the energy gap of the vertical heterojunction $(1.98 \mathrm{eV})$ in polyblends is lower than that of the pristine MEH-PPV (2.14 eV), which is believed to result in the improved EL properties.
\end{abstract}

\section{Introduction}

Poly(2-methoxy-5-(2'-ethyl-hexyloxy)-p-phenylenevinylene) (MEH-PPV) has attracted enormous attention recently because of its potential use in polymer light-emitting diodes (PLEDs) owing to low turn-on voltage, good solubility and high electroluminescence (EL) efficiency [1-4]. Many researchers attempted to improve its EL performance by removing the imperfections caused by polymerization through thermal treatments [5-7]. Besides, MEH-PPV is an orangered emissive polymer. Attempts to tune the color have frequently been made by copolymerization with other conjugation polymers [8-12].

Poly(2,3-diphenyl-5-octyl- $p$-phenylenevinylene) (DPOPPV) is a blue-green emitting polymer. We have successfully copolymerized it with MEH-PPV via the Gilch route [13] and found that the resulting copolymers tended to form an alternative copolymer [12]. Thus, the emitted color can be well tuned from blue-green to orange-red by adjusting the feed ratio of the monomers for copolymerization. However, when we employed the copolymers as a light-emitting layer to manufacture the PLED with indium tin oxide (ITO) as an anode, polyaniline (PANI) as a hole-transporting layer, and both $\mathrm{Ca}$ and $\mathrm{Al}$ metals as a composite cathode, their EL efficiencies are only between those of the pristine $\mathrm{MEH}-$ PPV and DPO-PPV [14]. No synergistic effect was found.

The energy gap between the lowest unoccupied molecular orbital (LUMO) and the highest occupied molecular orbital (HOMO) levels for DPO-PPV is $2.64 \mathrm{eV}$, whereas that for MEH-PPV is $2.14 \mathrm{eV}$ [14]. If the excitons are present in DPO-PPV chains that are adjacent to the MEHPPV chains, energy transfer from DPO-PPV to MEH-PPV would occur. If the exciton is present in MEH-PPV that is surrounded by DPO-PPV chains, there would be less chance of it being quenched. Therefore, in this study we blended MEH-PPV with DPO-PPV in their co-solvent toluene and investigated their miscibility in spin-cast films by fluorescence spectroscopy, differential scanning calorimetry and two-photon excitation microscopy. The possible synergistic effects for polyblends on photoluminescence (PL) and EL performances were also investigated.

\section{Experimental}

\section{Materials}

The monomer synthesis of MEH-PPV and DPO-PPV and their polymerizations via the Gilch route can be found elsewhere [12]. The weight-average molecular mass and polydispersity of the resulting MEH-PPV measured by gel permeation chromatography were $288,000 \mathrm{~g} / \mathrm{mol}$ and 3.02 , whereas those of DPO-PPV were $281,000 \mathrm{~g} / \mathrm{mol}$ and 3.87 , with reference to polystyrene (PS) standards. Their blends in various weight ratios were prepared as a film form by dissolving individual polymers in toluene at room temper- 
ature first, mixing two solutions thoroughly and then spincasting the mixed solutions onto a glass plate.

\section{Characterization}

UV-vis absorption spectra were recorded using a JASCO model 555 spectrometer. PL spectra were recorded with a SPEX 1403 fluorescence spectrophotometer using a xenon lamp as an excitation source. The two-photon excitation image of polyblends was investigated by using a Leica TCS SP2 confocal spectral microscope imaging system. The wavelength of the excitation source was $488 \mathrm{~nm}$, whereas that of the emitting light was collected in the range 500-700 $\mathrm{nm}$. Differential scanning calorimetry was performed under a nitrogen atmosphere in a TA Instruments model 2920 differential scanning calorimeter. Data were acquired from the second run to remove the thermal-aging effect. The first run was conducted by heating the specimens at a rate of $10{ }^{\circ} \mathrm{C} / \mathrm{min}$ from 50 to $300{ }^{\circ} \mathrm{C}$, retaining the specimens at $300{ }^{\circ} \mathrm{C}$ for $3 \mathrm{~min}$ and then quenching to $50^{\circ} \mathrm{C}$. The second run was conducted by repeating the heating schedule of first run as soon as the thermal equilibrium had been reached.

The PLED devices were fabricated by the following procedure. A $2 \times 3-\mathrm{cm}^{2}$ ITO-coated glass plate was patterned with $200 \times 200 \mu^{2}$ pixels. After thoroughly cleaning it and treating it with $\mathrm{O}_{2}$ plasma, it was spin-coated with a proprietary PANI solution as a hole-transporting layer. After baking it at approximately $60{ }^{\circ} \mathrm{C}$ to remove the solvent, MEH-PPV/DPO-PPV blending samples in toluene were spin-coated onto the PANI layer. After baking, $\mathrm{Ca}$ and Al metals were sequentially evaporated under high vacuum (approximately $10^{-6}$ Torr) to deposit them onto the polyblend layer as a composite cathode. The final configuration of the PLED device is designated as ITO/PANI/ polyblend/Ca/Al.

The current-voltage characteristics of the PLED devices prepared and the concurrent EL light intensities were measured in a pixel area with a Spectra Scan model PR705 spectraradiometer. The EL spectra of the devices were recorded with a SPEX1403 fluorescence spectrophotometer at a forward bias of $15 \mathrm{~V}$.

\section{Results and discussion}

\section{PL properties and miscibility}

UV-vis absorption spectra of MEH-PPV/DPO-PPV blending samples with various weight ratios were shown in Figure 1. The onset absorption wavelength $(\lambda)$ of MEHPPV is $580 \mathrm{~nm}$, which is consistent with the data reported in the literature $[8,15,16]$. As the content of DPO-PPV was over $50 \mathrm{wt} \% \%$, the absorption of DPO-PPV became significant at $\lambda<450 \mathrm{~nm}$ (Figure 1a). Notably, the absorption of MEH-PPV was persistently detectable even when its content was only $1 \mathrm{wt} . \%$ (Figure $1 \mathrm{~b}$ ).

Figure 2 shows the PL spectra of MEH-PPV/DPO-PPV blending samples with various weight ratios excited at a (a)

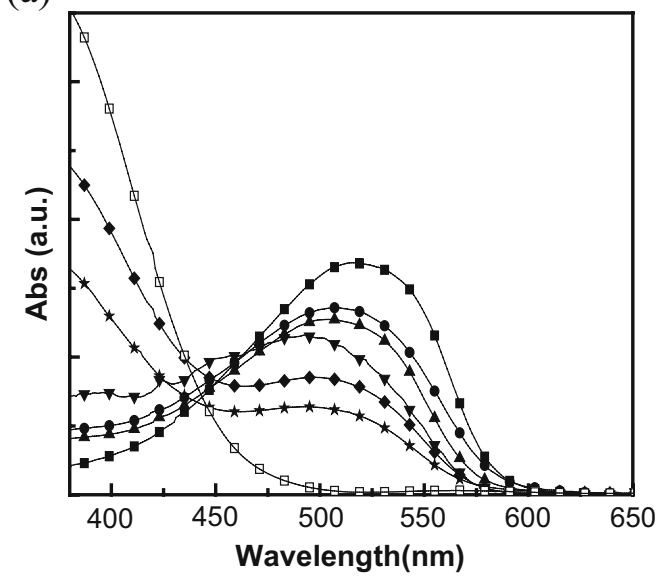

(b)

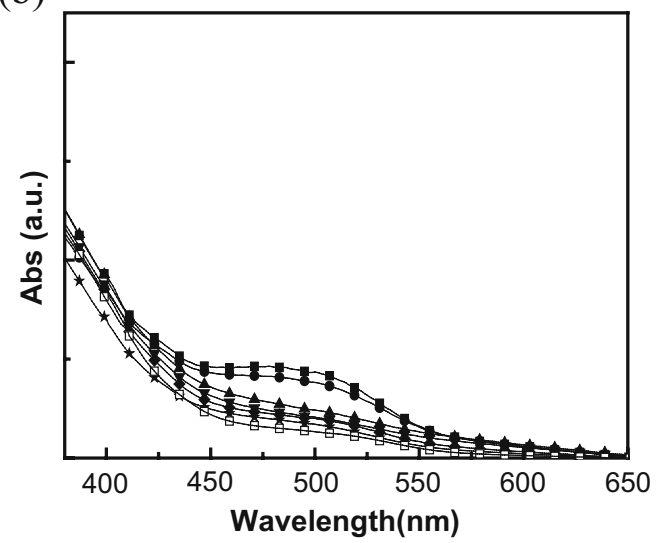

Figure 1. UV spectra of various MEH-PPV/DPO-PPV polyblends (a) with ratios of $(\boldsymbol{\square}) 1: 0,(\bullet) 6: 1,(\mathbf{\Delta}) 3: 1,(\boldsymbol{\nabla}) 1: 1,(\bullet) 1: 3,(\star) 1: 6$, (ㅁ) $0: 1$; and (b) with ratios of $(\boldsymbol{\square}) 1: 50,(\bullet) 1: 55,(\mathbf{\Delta}) 1: 60,(\boldsymbol{\nabla}) 1:$ $65,(\diamond) 1: 70,(\star) 1: 75,(\square) 1: 100$.

wavelength of $400 \mathrm{~nm}$. MEH-PPV has a main emission peak at $590 \mathrm{~nm}$ and a shoulder at $630 \mathrm{~nm}$. The latter is generally agreed to be contributed by the chain aggregations [17-19]. DPO-PPV has an emission peak at $510 \mathrm{~nm}$, which is within the absorption range of MEH-PPV. According to our previous studies on the energy transfer between donor-acceptor systems for the luminescent properties of optoelectronic polymers, the effective distance between the donors and acceptors for energy transfer is up to $50 \AA[20,21]$. Above that distance, the energy transfer efficiency $\phi_{\mathrm{ET}}$ decreases rapidly with the distance $r$ as suggested by the following equation [22]:

$$
\phi_{\mathrm{ET}}=\frac{R_{\mathrm{o}}^{6}}{R_{\mathrm{o}}^{6}+r^{6 \prime}}
$$

where $R_{\mathrm{O}}$ is the Förster distance defined as the distance at which the transfer rate is equal to the decay rate of the donor in the absence of the acceptor.

Because in the MEH-PPV/DPO-PPV polyblends the DPO-PPV emission persistently appeared at $510 \mathrm{~nm}$ in the entire range of compositions under study, the energy transfer between the two polymers was apparently insufficient, indicating that they were basically immiscible. DPOPPV formed a green domain by phase separation as revealed by two-photon excitation microscopy of the 
(a)

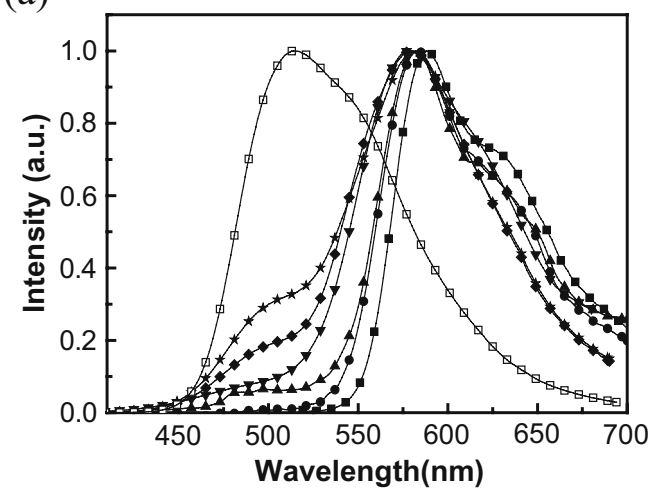

(b)

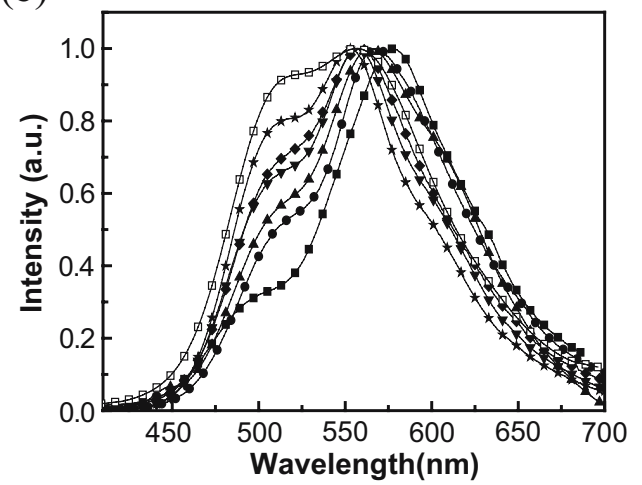

Figure 2. PL spectra of various MEH-PPV/DPO-PPV polyblends; (a) with ratios of $(\bullet) 1: 0,(\bullet) 6: 1,(\mathbf{\bullet}) 3: 1,(\boldsymbol{\nabla}) 1: 1,(\bullet) 1: 3,(\star) 1: 6$, (ㅁ) $0: 1$; and (b) with ratios of (ष) $1: 50,(\bullet) 1: 55,(\boldsymbol{\bullet}) 1: 60,(\boldsymbol{\nabla}) 1:$ $65,(\star) 1: 70,(\star) 1: 75,(\square) 1: 100$

1:1 polyblend (Figure 3). DPO-PPV is a crystal-liable polymer with a melting temperature of $285^{\circ} \mathrm{C}$ and a glasstransition temperature of $123{ }^{\circ} \mathrm{C}$ (Figure 4). MEH-PPV has a glass-transition temperature of $65^{\circ} \mathrm{C}[23,24]$ and a broad transition peak at $230^{\circ} \mathrm{C}$ that has been associated with supramolecular nanosized aggregations [25]. The melting temperature of DPO-PPV was barely changed although its content in the polyblend was decreased from 100 to 50 wt.\%. It confirmed that MEH-PPV and DPO-PPV are basically immiscible.

The solubility parameters of toluene solvent, MEH-PPV and DPO-PPV are 18.21, 20.38, and $22.93\left(\mathrm{~J} / \mathrm{cm}^{3}\right)^{1 / 2}$, respectively, estimated by the group contribution method.

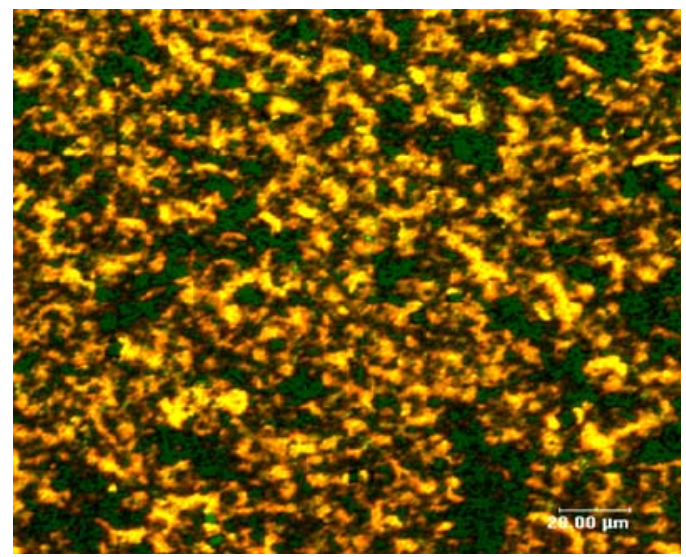

Figure 3. Two-photo excitation micrograph of MEH-PPV/DPO-PPV polyblend with $1: 1$ weight ratio.

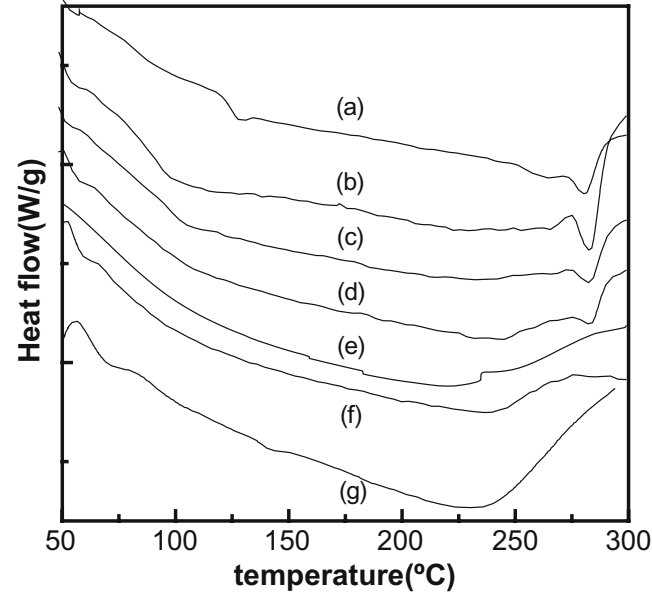

Figure 4. DSC plots of various MEH-PPV/DPO-PPV polyblends with following weight ratios: (a) $0: 1$, (b) $1: 6$, (c) $1: 3$, (d) $1: 1$, (e) $3: 1$, (f) $6:$ $1,(\mathrm{~g}) 1: 0$.

[26]. Thus, toluene is a better solvent for MEH-PPV than for DPO-PPV. As the solvent evaporates, a DPO-PPV-rich phase will form a domain dispersing in the more soluble MEH-PPV-rich phase first. A vertically segregated structure was thus formed in a spin-cast film as observed by two-photon excitation microscopy. Similar phase separation in spin-cast polyfluorene-based polyblends consisting of poly(2,7-(9,9-di- $n$-octylfluorene-alt-benzothiadiazole)) (F8BT) and poly(2,7-(9,9-di-n-octylfluorene)-alt-( $p$-phenylene-((4-sec-butylphenyl)imino)- $p$-phenylene) (TFB) using $p$-xylene as a solvent was reported [27]. The less soluble F8BT-rich phase was found to form a domain dispersing in the more soluble TFB-rich phase. However, as the content of MEH-PPV was too low to form a MEHPPV-rich continuous phase, phase inversion occurred and the DPO-PPV-rich phase became a continuous phase.

\section{EL properties}

EL intensity-voltage characteristics of the ITO/PANI/ polyblend/Ca/Al PLED devices are present in Figure 5. The turn-on voltage for the PLED prepared with pristine MEH-PPV was $2.8 \mathrm{~V}$, whereas those prepared with the polyblends were in the range $1-2.6 \mathrm{~V}$. MEH-PPV has a HOMO level at $-5.04 \mathrm{eV}$ and a LUMO level at $-2.9 \mathrm{eV}$, whereas DPO-PPV has a HOMO level at $-5.7 \mathrm{eV}$ and a LUMO level at $-3.06 \mathrm{eV}$ [14]. If MEH-PPV acts as a hole-transport polymer and DPO-PPV acts as an electrontransport polymer, the energy gap of the heterojunction would be $1.98 \mathrm{eV}$, lower than that of the pristine MEHPPV. Thus, the lower turn-on voltage for the polyblends might be due to the so-called "type II" heterojunction [27, 28] with lower interfacial energy gap between the HOMO level for the hole-transport MEH-PPV and the LUMO level for the electron-transport DPO-PPV. It is highly possible because the MEH-PPV-rich layer is a wetting layer directly coated on top of the hole-injecting PANI layer and DPOPPV forms a domain dispersing on top of the MEH-PPVrich layer during phase separation. However, why did we not observe the PL and EL emissions from the exciplexes 
formed in the heterojunctions? The answer to that question is that the barrier for the exciplex to move away from the heterojunction is only $160 \mathrm{meV}$. The thermal energy of the exciplex at room temperature should be able to overcome this barrier, so only efficient bulk exciton emission was observed. This phenomenon has been well studied by Morteani et al. [28] and other research groups [29, 30].

As to the EL light intensity, the PLED prepared with pristine MEH-PPV emitted $190 \mathrm{~cd} / \mathrm{m}^{2}$ at $14 \mathrm{~V}$, a much higher intensity than that prepared with DPO-PPV $(22 \mathrm{~cd} /$ $\mathrm{m}^{2}$ ). It should be noted here that our data were obtained from measurements on a single pixel of $200 \times 200-\mu \mathrm{m}^{2}$ area of PLED specimens. Because no stray light was counted, the measured EL light intensity of pristine $\mathrm{MEH}-$ PPV was slightly lower than other data reported for MEHPPV [31]. It is interesting to find that the polyblends had an EL emission intensity higher than for both pristine polymers at $14 \mathrm{~V}$ even when they contained only $1 \mathrm{wt} . \%$ of MEH-PPV. The highest EL light intensity is $850 \mathrm{~cd} / \mathrm{m}^{2}$ at $16 \mathrm{~V}$ for the polyblend containing 75 wt. $\%$ of $\mathrm{MEH}-$ PPV.

As to the EL quantum yields, all the polyblends had higher yields than both pristine MEH-PPV and DPO-PPV as shown in Figure 6. Although the polyblend with an MEH-PPV to DPO-PPV weight ratio of 1:6 has the highest quantum yield with low current, the quantum yield decreased with the current from 5.48 to $0.577 \mathrm{~cd} / \mathrm{A}$ and finally less than that with a 3:1 ratio. The quantum yield of the latter slightly increased with the current and reached $0.654 \mathrm{~cd} / \mathrm{A}$. However, most of the polyblends showed a decrease of EL quantum yields with current density, indicating that the blending systems are rather unstable at high current density. Furthermore, because the dispersion of DPO-PPV in the blending system was still poor as shown in Figure 3, the heterojunction effect did not fully contribute to the EL emission. In a subsequent study, we have provided the chemical bonding between two mixed MEH-PPV/DPO-PPV polymers by thermal treatment and found an at least threefold increase of EL intensity and quantum yield compared with the untreated polyblend (K.F. Lin, Y.L. Fan and H.-L. Chou, unpublished results).

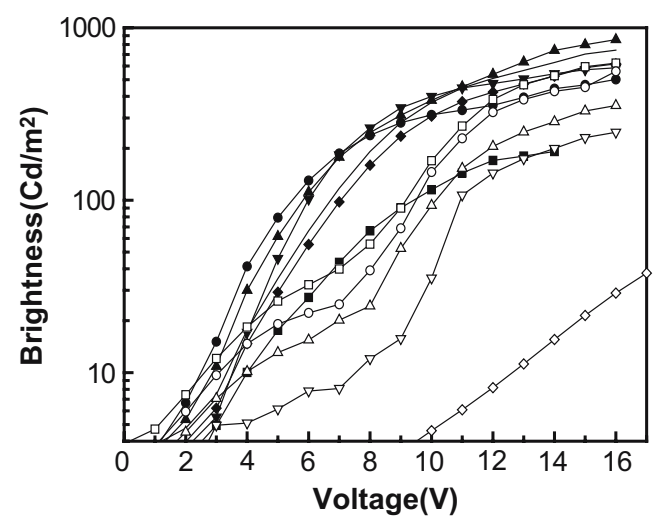

Figure 5. L-V plots of PLED prepared by various MEH-PPV/DPO-PPV polyblends with following weight ratios: $(\boldsymbol{\bullet}) 1: 0,(\bullet) 6: 1,(\mathbf{\Delta}) 3: 1$,

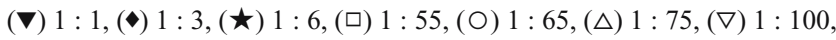
$(\diamond) 0: 1$.

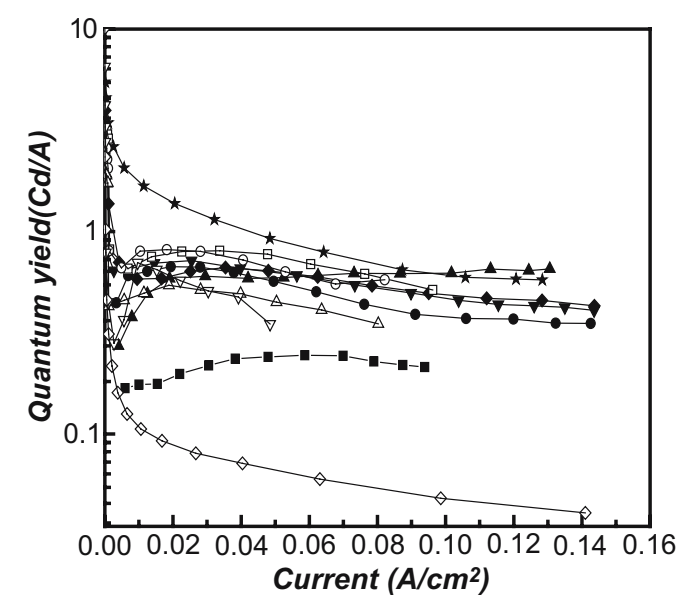

Figure 6. Current dependence of EL quantum yield of PLED prepared by various MEH-PPV/DPO-PPV polyblends with following weight ratios: (匹) $1: 0,(\bullet) 6: 1,(\boldsymbol{\Delta}) 3: 1,(\boldsymbol{\nabla}) 1: 1,(\diamond) 1: 3,(\star) 1: 6,(\square) 1: 55,(\bigcirc)$ $1: 65,(\triangle) 1: 75,(\nabla) 1: 100,(\diamond) 0: 1$.

Figure 7 shows the EL spectra of MEH-PPV, DPO-PPV and their blends at a forward bias of $15 \mathrm{~V}$. The EL spectra of pristine MEH-PPV and DPO-PPV are similar to their PL spectrum. The main peak of the EL spectrum of MEH-PPV is at $580 \mathrm{~nm}$, whereas that of DPO-PPV is at $480 \mathrm{~nm}$; however, the EL spectra of the polybends are blueshifted with increasing content of DPO-PPV, the behavior of which is different from that of the PL spectra (Figure 2). The blueshift of the EL spectra can be divided into two stages. As the content of DPO-PPV was first increased to 50 wt.\%, the spectra were only slightly blueshifted until 75 wt.\% was reached. The EL spectrum of polyblends with 75 wt.\% of DPO-PPV was rather broad, covering from 480 to $680 \mathrm{~nm}$. We believe that the MEH-PPV-rich phase and the DPO-PPV-rich phase in this weight ratio were intermingled into a co-continuous phase. As the content of DPO-PPV was further increased to 98.6 wt.\% (75:1 weight ratio), the left part of the EL spectrum was barely changed but its right part was significantly blueshifted, indicating that the MEH-PPV-rich phase diminished.

According to the EL spectra, MEH-PPV apparently dominated the EL emission of the polyblends. However,

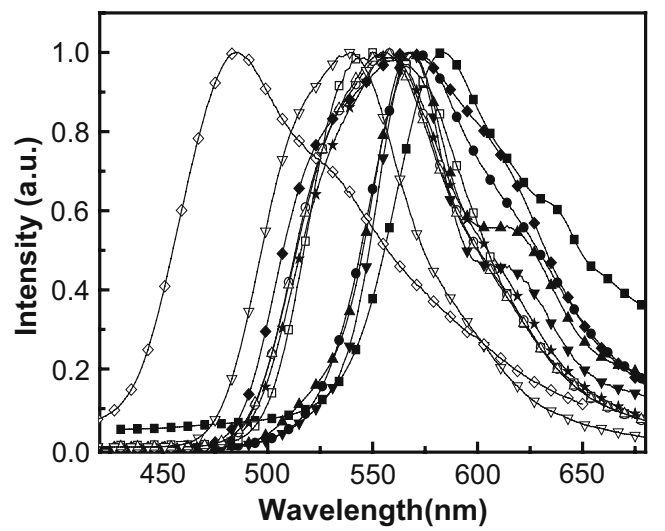

Figure 7. EL spectra at a forward bias of $15 \mathrm{~V}$ for MEH-PPV/DPO-PPV polyblends with following weight ratios: (⿴) $1: 0,(\bullet) 6: 1,(\mathbf{\Delta}) 3: 1,(\boldsymbol{\nabla})$ $1: 1,(\diamond) 1: 3,(\star) 1: 6,(\square) 1: 55,(\bigcirc) 1: 65,(\triangle) 1: 75,(\nabla) 1: 100,(\diamond)$ $0: 1$. 


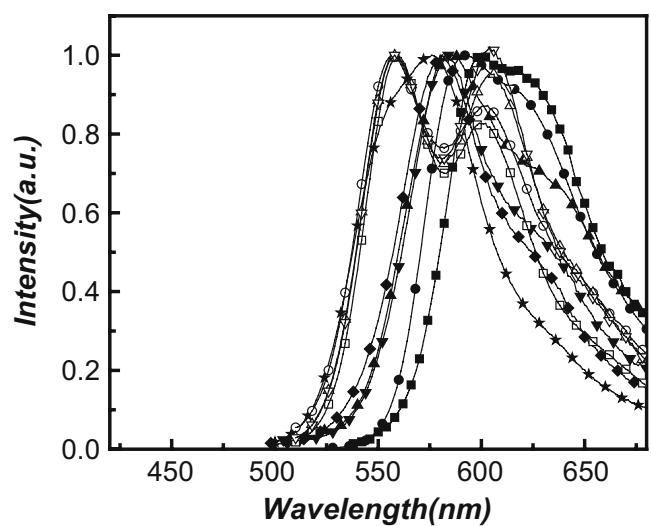

Figure 8. PL spectra of various MEH-PPV/PS polyblends with following weight ratios: (■) $1: 0,(\bullet) 6: 1,(\boldsymbol{\Delta}) 3: 1,(\nabla) 1: 1,(\diamond) 1: 3,(\star) 1: 6$, (ㅁ) $1: 55,(\bigcirc) 1: 65,(\triangle) 1: 75,(\nabla) 1: 100$.

the question is whether DPO-PPV segments directly contribute to the EL emission. To answer this question, we used a nonemissive PS to mix with MEH-PPV in toluene and spin-coated the MEH-PPV/PS solutions on a glass plate. The PL spectra of so-formed MEH-PPV/PS blending films are shown in Figure 8. As the content of PS was increased, the PL spectrum was blueshifted. The extent of the blueshift is similar to that of the EL spectra of MEHPPV/DPO-PPV films (Figure 7). Apparently, the majority of the EL emission was from the MEH-PPV segments and the DPO-PPV chains only played a supporting role to enhance the emission of MEH-PPV. The blueshift of the EL spectrum was mainly due to the disaggregation of MEH-PPV chains.

Finally, the increased EL efficiency for MEH-PPV/ DPO-PPV polyblends compared with the EL efficiency of the individual components was believed to be attributed to the following two aspects: one is the formation of a vertically segregated structure with the type II heterojunction that created a well-defined capture zone for charges so as to reduce the leakage current [32]; the other is owing to the fact that DPO-PPV has a higher energy gap than MEHPPV. If the excitons are present in DPO-PPV chain segments that are adjacent to the MEH-PPV chain segments, energy transfer from DPO-PPV to MEH-PPV would occur. If the exciton is present in MEH-PPV that is surrounded by the DPO-PPV chain segments, there would be less chance of it being quenched. This aspect is important as the content of MEH-PPV was less than 2 wt.\%, so MEH-PPV might dissolve into the DPO-PPV domains.

\section{Conclusions}

MEH-PPV and DPO-PPV are basically immiscible, rendering insufficient energy transfer from DPO-PPV to $\mathrm{MEH}-\mathrm{PPV}$ in PL of spin-cast films from their solutions in toluene. Because toluene is a better solvent for MEH-PPV than for DPO-PPV, the MEH-PPV-rich layer formed a wetting layer and DPO-PPV-rich domains formed a dispersive phase. Consequently, a lower turn-on voltage for the PLED prepared compared with that of the pristine
MEH-PPV was observed and was attributed to the type II heterojunction between the hole-transport MEH-PPV segments and the electron-transport DPO-PPV segments. All the polyblends had higher EL quantum yields than both pristine MEH-PPV and DPO-PPV. Although the EL spectrum of the polyblend was blueshifted with increasing content of DPO-PPV, the majority of the EL emission was from the MEH-PPV segments. DPO-PPV chains only played a supporting role to enhance the emission of MEHPPV.

\section{Acknowledgements}

The authors acknowledge financial support of the National Science Council of Taiwan, Republic of China, through grant NSC91-2216-E002-004 and the National Economic Ministry of Taiwan, Republic of China, through grant 92EC-17-A-08-S1-0015.

\section{References}

1. D. Braun and A. Heeger, J. Appl. Phys. Lett., 58, 1982 (1991).

2. I. D. Parker, J. Appl. Phys., 75, 1656 (1994).

3. G. Padmanaban and S. Ramakrishnan, J. Am. Chem. Soc., 122, 2244 (2000).

4. Y.-L. Fan and K.-F. Lin, J. Polym. Sci. Part A: Polym. Chem., 43, 2520 (2005).

5. T. W. Lee and O. O. Park, Adv. Mater., 12, 801 (2000).

6. T. W. Lee, O. O. Park, L. M. Do and T. Zyung, Synth. Met., 117, 249 (2001).

7. H.-L. Chou, K.-F. Lin, Y.-L. Fan and D.-C. Wang, J. Polym. Sci. Part B: Polym. Phys., 43, 1705 (2005).

8. B.-H. Sohn, K. Kim, D. S. Doi, Y. K. Kim, S. C. Jeoung and J.-L. Jin, Macromolecules, 35, 2876 (2002).

9. L. S. Park, Y. S. Han, S. D. Kim and D. U. Kim, Synth. Met., 117, 237 (2001).

10. Y. Xiao, W.-L. Yu, Z. Chen, W. Huang and A. J. Heeger, Synth. Met., 106165 (1999).

11. S. M. Chang, P. K. Su, G. J. Lin and T. J. Wang, Synth. Met., 137, 1025 (2003).

12. C.-C. Chiu, K.-F. Lin and H.-L. Chou, J. Polym. Sci. Part A: Polym. Chem., 41, 2180 (2003).

13. H. G. Gilch and W. L. Wheelwright, J. Polym. Sci. Part A: Polym. Chem., 4, 1337 (1966)

14. H.-L. Chow, K.-F. Lin, Y.-K. Han and D.-C. Wang, Bull. Coll. Eng $N T U, \mathbf{8 9}, 111$ (2003).

15. Y. Li, Y. Cao, J. Gao, D. Wang, G. Yu and A. J. Heeger, Synth. Met 99, 243 (1999).

16. K. M. Gaab, and C. J. Bardeen, J. Phys. Chem. B, 108, 4619 (2004).

17. T. Q. Nguyen, R. Y. Yee and B. J. Schwartz, J. Photochem. Photobiol. A, 144, 21 (2001).

18. T. Huser and M. Yan, Synth. Met., 116, 333 (2001).

19. T. Q. Nguyen, B. J. Schwartz, R. D. Schaller, J. C. Johnson, L. F. Lee, L. H. Haber and R. J. Saykally, J. Phys. Chem. B, 105, 5153 (2001).

20. H.-L. Cheng and K.-F. Lin, Synth. Met., 122, 387 (2001).

21. H.-L. Cheng and K.-F. Lin, J. Mater. Chem., 12, 2270 (2002).

22. J. R. Lakowicz, Principles of Fluorescence Spectroscopy. Plenum, New York, 1983, Chap. 3.

23. Y. Liu, M. S. Liu, X. C. Li and A. K. Y. Jen, Chem. Mater., 10, 3301 (1998).

24. T. W. Lee and O. O. Park, Adv. Mater., 12, 801 (2000).

25. S.-H. Chen, A.-C. Su, Y.-F. Huang, C.-H. Su, G.-Y. Peng and S.-A. Chen, Macromolecules, 35, 4229 (2002). 
26. D. W. Van Krevelen, Properties of Polymers: Their Correlation with Chemical Structure: Their Numerical Estimation and Prediction from Additive Group Contributions. Elsevier, Amsterdam, 1990.

27. J.-S. Kim, P. K. H. Ho, C. E. Murphy and R. H. Friend, Macromolecules, 37, 2861 (2004).

28. A. C. Morteani, A. S. Dhoot, J.-S. Kim, C. Silva, N. C. Greenham, C. Murphy, E. Moons, S. Cina, J. H. Burroughes and R. H. Friend, Adv. Mater., 15, 1708 (2003).
29. R. Jakubiak, C. J. Collison, W. C. Wan, L. J. Rothberg and B. R. Hsieh, J. Phys. Chem. A 103, 2394 (1999).

30. M. Lor, J. Thielemans, L. Viaene, M. Cotlet, J. Hofkens, T, Weil, C. Hampel, K. Müllen, J. W. Verhoeven, M. Van der Auweraer and F. C. De Schryver, J. Am. Chem. Soc., 124, 9918 (2002).

31. I. D. Parker, J. Appl. Phys., 75, 1656 (1994).

32. A. C. Morteani, R. H. Fiend and C. Silva, Chem. Phys. Lett., 391, 81 (2004). 